\title{
Do we know what a body can do? \#1
}

\author{
Interview
}

Arno BöHLeR AND ERIN MANNING

ARNo BöHLER: Spinoza's question „Do we know what a body can do?“ became a major research issue in your workshop with Brian Massumi at Tanzquartier Vienna yesterday. Do we know what a body can do?

ERIN ManNING: For me, what a body can do has been a key question for the past decade. My own approach to the question has been to move toward the prelinguistic and ask how movement activates a body in the midst of a process of becoming. I call this bodying. Working through a lexicon of movement in its preacceleration - before displacement as such - I have wanted to explore how movement activates a modality of thought that is of the becoming-body. Such a thinking cannot be directly articulated in language, and yet it is a thinking (or a thinking-feeling) in its own right. A thinking in the moving. What's interesting about this is that it moves thought from a category of organization toward an expression of difference. Thought in the moving is the activation of a differential. It is the making-felt of a changing relationscape. So, I suppose the first answer I could give is that what a body can do is think, in the moving.

I spend a lot of time watching bodies move - in the subway, on the street, in the classroom, in the studio. And I ask myself two questions: what makes us so certain that thinking is not in-the-moving, or in-the-feeling; and what makes us so certain that we can define a body in time and space as a separate and individual entity? These are old cartesian categories, the first putting thought in the mind, out of the body; the second placing the body outside of its relation to the world. Whatever makes it possible for us to think of a body as defined apart from its participation in the world is the same thing that allows us to believe that thought can be contained by language, apart from the movements that generate it. In my account of what a body can do, I therefore begin with an attention to the question of the "in act" of the doing. This emphasizes the microgestures of 
becoming that are in the bodying. These microgestures are a thinking-in-theact, I argue, not in the sense that they resolve into this or that conscious thought, but in the sense that they are replete with intensity and directionality. Bodying does resolve into this or that form, eventually, and repeatedly, but it does not begin there, nor does it remain there. A body is the metastability of those microgestures more than it is a form as such. If we begin there, with the microgesture, it becomes clear that a body is more associated milieu (Simondon) than form. What a body does is ecological: it becomes in relation to a changing environment, and what it does in that relation is what it is. A body is a tending, an inflection, an incipient directionality. And this incipiency includes a thinking in its own right.

Of course, what a body does always has a place and a time. People often ask how such an account of the body has agency (this question is often allied to the thinking of gender and race). I prefer the notion of "agencement" to agency the sense of directionality occasioned by movement rather than a subject-based intentionality - but however you define this moment of "making a difference," there is no question that how it individuates in this time and place, in co-composition - or how it matters, here and now - belongs to what a body can do. A body makes a difference in terms of how this or that vector, this or that inflection, alters the conditions of this or that event. So, that a body is black or white or female or transgender does make a difference. Of course it does! But these are less "states" of an existing body than vectors of a becoming-body that themselves change over time. Identity, like individuation, is emergent. What a body can do is change.

ARNo BöHLer: So you suggest that bodies envelop a certain field which we and other bodies already share. This means that one cannot separate the question of what a body can do from the milieu in which it dwells in relation with others. During your workshop with Brian you presented a beautiful picture: What happens when there is light coming through a window and it affects you? Being in touch with the sun is more than being a subject contemplating an external object. Are we becoming sunlike in such a moment?

ERIN MANNing: Yes, that's a beautiful way of putting it. As an artist, I have always been very interested in these kinds of threshold effects. Like that moment when you are sitting at a café and you have been in an intense conversation and suddenly you look up and you notice that it got dark, but you cannot say when the light changed. And yet, upon noticing the shift in light you immediately know 
that the changing conditions affected how the conversation progressed. Threshold effects make a difference, and yet they are rarely experienced as such.

ARNo BöHLER: Would this not mean that bodies are worldwide entities? Singularities of a manifold, worldwide field, shared not only with other human bodies but with innumerous non-human bodies as well? Like our liaisons with the sun, for instance? An intimate relationship between our human body and a non-human body? Or the special liaison of plants with the sun?

ERIN Manning: William James said that the relations are as real as the terms of the relation. That is how he defines radical empiricism. So yes, the relationscape of the bodying is worldwide, even otherworldy, in the sense that it invents worlds. A body is a field of relation out of which and through which worldings occur and evolve. We know neither where a world begins nor where a body ends. What is real, what we know, is relations. This is speculative pragmatism: relations are real, here and now, but what they can do is unknowable in advance, must continuously be invented.

What is at stake in the field of relation is how the relation evolves, how it expresses itself, what it becomes, what it can do. The relation can never be properly called human. It may pass through the human or connect to certain human tendencies, but in and of itself it is always more-than-human.

So, the question of the non-human or the more-than-human is of central importance as regards what a body can do. There must be a way to conceive of a bodying that begins and returns to the midst, to the relational field that is morethan-human. A focus on the middling of experience leads us toward a modality of thinking the becoming body in a directly ecological sense in terms of an ecology of practices that includes the human but is not limited to the human.

One of the ways in which I have tried to address this directly ecological experience of the world is through what I have called "autistic perception." I define autistic perception as a direct experience of emergent field effects. When autistics first perceive, they do not tend to see forms as non-autistics do. Instead, they see perception's very emergence: edges, shadows, colors, shapes. What takes a half second for a neurotypical to perceive as form can take up to five minutes for an autistic. This lag in the taking of form allows autistics to perceptually dwell in the shape-shifting of experience and gives them a lived sense of the malleability of form. This is not completely outside neurotypical experience, simply less often directly experienced - artists hone such perceptual tendencies, and they are not unfamiliar with those who drink or take drugs, or those who expe- 
rience extreme states due to shock or love or fear. However we come to autistic perception, what is important, I think, is the accounting of a phase of experience where it is the associated milieu of relation which is at the forefront not only conceptually but perceptually. Were we to honour such a perceptual account of process, I think we might come to see the everyday quite differently. This would then have important effects not only on our experience of the world but on what we do collectively, politically.

To experience the world in its shape-shifting alerts us to the realness of relation and connects to a more-than-human horizon, I think. It allows us to think ecologically, to begin in the middle. And from there, there is an opening to the felt expression of thought-in-the-moving, to language's prelinguistic expressions - what I have elsewhere called prearticulation - to the complex rhythms of what lies between the conscious and the non-conscious at the interstices of the human and the nonhuman, to the more-than-human.

ARNo BöHLER: So if I've got you right, it is not enough to speak only in the name of the other things, but really to change, or to open up a space in which another form of relationality with other non-human things becomes possible. So, it is not only a matter of language and a matter of morality to take care of other non-human things and give them a voice, but to work on the level of affection, of affect, of being affected, of what you and Brian also called tendencies which show up in relation to others and to change our ways of behavior. Or, as you've expressed it so beautifully, to care for the event.

Erin Manning: Yes, I think that there can be a tendency when we talk about the non-human to assume we are talking about the animal or the vegetal, which we are not doing at all. When you speak about the field, what you are taking into account is also the more-than-human of the animal, the more-than-human of the oxygen, the more-than-human of the human. In my recent work I call this speciation, as opposed to species. Relational fields rather than categories. Emergent constellations. A constellation meets a constellation rather than the particularity meeting a particularity. If you begin to think in terms of that kind of multiplicity, then, immediately I think, there is that sense of the care for the event, because the constellation is already eventful. As Whitehead would say, an event always carries with it concern for its eventness. Self-enjoyment of the event, in the event. This is punctual, of course. A constellation or speciation is emergent here and now, this or that way. Pragmatic, yet speculative. 
One way I think about the care for the event in the event is in terms of what I call the dance of attention. Not human attention, but field attention - the event's attention to its own development, its own concrescence. There is very little in our everyday lives that facilitates this kind of dance of attention. In the performing world we do sometimes experience this dance of attention. When it is strong, that's when we leave a performance and say: "Wow, that really worked!" What is felt in such instances is the lived intensity of the event's capacity to create a field of experience. But in the everyday it seems to me that we need to constantly develop techniques that allow for that dance of attention to be awakened or energized, to be made possible. It is there, it is latently there, I think, all the time. But it can be overcoded by the habitual tendencies of subject-centered intentionality that work continuously to take us out of the field of relation and into the individual, as though the individual could be cordoned out from the event. The techniques to be invented are ones that open the perceptual register beyond intentionality to the thought-in-the-moving of the complex emergent patterns of our daily lives.

ARNo BöHLER: If we take bodies as world-wide entities, the global field, which they share, becomes one of the most important features of any body. Deleuze even speaks of a transcendental field, the all-one world which any body shares with all others by populating it.

Erin Manning: I think of this as worlding and it reminds me of the necessity to consider the body always from the perspective of the collective, or the transindividual. I am thinking of an example here. When we were thinking about the body and what the body can do, I wanted to give an example from disability culture. In disability activism it is often said that disability is not what happens to the body, it is not about the state of the body. Disability is about a culture that does not accommodate for diversity. So, the body is not disabled, the culture is disabled in its incapacity to create accommodations that allow for difference different kinds of bodying - to exist.

I am thinking about a disability-activist, Harriet McBryde-Johnson, a lawyer. She once was invited by the New York Times to do a spread on her work for "Not Dead Yet", which is an organization that is very much against euthanasia and assisted suicide. At first, when I was reading Harriet McBryde-Johnson as part of my ongoing research into disability politics, it was to better understand why it was that so many disability activists felt so strongly against assisted suicide. Because, from my perspective, it seemed that what a body can do is also choose 
to die. What I learned through that reading was that not every body is given the same right to live. A disabled body is often a body seen by the medical establishment as not worthy of life. So a disabled person who has cancer doesn't necessarily get the same kind of care as a non-disabled person. This happens in subtle ways - for instance a doctor might be quicker to speak to the disabled person about palliative care, assuming that their quality of life is already jeopardized by their disability and that the further strain of treatment may not be worthwhile. This example, which comes up very often in disability activist blogs (see, for instance, Bad Cripple), emphasizes the way disability is taken as an individual rather than as a collective, cultural problem, an ecology of practices. To turn this around it is necessary to keep asking not how this or that body performs but how this particular field of relation opens itself to the complexity of bodying. In the case of disability, too often an individual normative standard is imposed that presumes abled-bodiness as the very definition of a body, ignoring the complexity of evolution within all bodyings.

Lately I've been trying to understand accommodation within this context of transindividual bodying. Within disability politics, there tends to be an understanding that it is the lack of accommodation that creates the disability. This is no doubt true and needs to change: there needs to be a general understanding that making our worlds accessible to difference is to enrich them. But I want to push the term even further beyond the able-bodied/disabled divide, always keeping in mind that the person is not the one with the disability but that it is rather the lack of accommodation in the everyday environment that creates the rift between the able and the disabled, thus creating a culture of disability. Everywhere I turn within the complex work that unfolds within disability activism, I find complex reformulations of what it might mean to be accommodated. On the surface, the concerns can be very pragmatic: why is it that the tables that "accommodate" wheelchairs in a restaurant tend to be in the worst possible places (beside the bathroom, out of sight), why is it that "accommodating" entrances are in the back of hotels or in the bowels of buildings rather than out there in the open? Why isn't every entrance designed to accommodate difference, and why is it that there is a sense, still very strong within architecture (and within so many of our practices) that accommodation is a nasty afterthought? It seems to me that the question also goes deeper, touching on what is imaginable (thinkable, perceivable) within the field of experience - I wonder here, for instance, about the way in which thresholds between rooms become very apparent if you're in a wheelchair, or if you're blind, but otherwise may not "appear" in experience, or the way air moves sound in a room where the windows are open as opposed to 
where the windows are closed. I wonder whether there might be a way to take back the term "accommodation" to speak about all becoming-bodies, human and more-than-human, thereby opening up the question beyond the issue of the able and the disabled. Is there any event that does not require accommodation? Are we willing to think of what a body can do in a way that takes seriously how the event accommodates its shifting ecologies?

What would it mean to become aware, for instance, of how a lack of accommodation impedes the dance of attention of an event from being felt? What might it mean to make accommodation, seen as a collective engagement that tends fields of relation, a central concern of the everyday, in all instances, at all times, including accommodation in the strictest physical, architectural sense, but also accommodation to neurodiverse forms of perception and attention? Lack of accommodation has to do with the real lack of imagination as well as an ignorance of the complexity of differentuals at the heart of speciations.

Accommodation understood this way is not an individual concern. It is a care for the event in its modulation. How the event accommodates is not separate from what a body can do.

Some people might say that this sounds utopian. I don't actually think that we are talking about something utopian. The seeds for this are everywhere present. This already happens in pockets of existence, it happens in the corners. Sometimes quite obviously. Sometimes much less so. The trick is, I think, to make those corners matter.

ARNo BöHLER: You founded a SenseLab to make the care of the event matter? What kind of territory, what kind of accommodation, what kind of hospitality, of care, cura, curie and courting takes place there?

ERIN Manning: You touched on the core concept of the SenseLab, which is hospitality. But it isn't an inter-subjective hospitality, as you pointed out. It is an eventbased hospitality. To make a thought-experiment, an action-experiment like the SenseLab, the event has to be hospitable to learning, to thinking, and to the collaborative gesture that feeds both.

Ten years into it, what I can say is that the event-based hospitality at the heart of our collective practice has made it possible for us to engender a culture of affirmation. I hadn't really seen it this way until I heard a SenseLabber explain to someone else that what we do at the SenseLab is say YES! I loved this description and wondered how this practice of saying yes has fed our modes of collaboration, allowing us to develop ways of collective decision-making that are not based in a 
kind of Habermasian consensual politics. Thinking it through, it became clear to me that creating an affirmative practice doesn't mean simply saying "yes, I follow you through every aspect of this idea." It means "yes, we'll try this and collectively hone the effects." The question thus moves beyond how this or that is a "good" idea to what the idea can do in relation to the event with which it cocomposes and the selection of what works itself becomes emergent - it emerges from the event ecology. This means that there is very little sense of who "owns" the idea. The idea becomes a proposition in the Whiteheadian sense: it does something, and that something has effects. If what it does invigorates, intensifies, becomes hospitable to the other event-based inflexions and tendencies, the idea takes hold. If not, that given idea simply tends not to be developed then and there. But ideas do get re-taken up later, suddenly re-emerging in new contexts, often brought forward by different people than those who might originally have offered them up. What I like about this approach - an approach that emerged organically over time - is that it is the event-potential of the idea that gets taken up rather than the individual's stake in it.

This allows for forms of collaboration that emerge at the level of the incipiency of thought - often we can't remember whose idea it was in the first place. This affirmative stance allows us, I think, to be sensitive to the demands of the event in its unfolding while also collectively orienting it. This is complex because we are so accustomed to working with the competing tendency wherein we separate the individual subject out of the event, assuming an individual intentionality. At the SenseLab we've been able to sidestep this tendency for the most part by using the event as a training ground for the invention of techniques coming out of a collective experimentation with the idea as affirmative proposition. Our process is mould an idea into a technique that can affect the conditions of the event, altering how the event comes to expression, affecting its dance of attention. Techniques we develop usually center on the event's thresholds - the moving across registers of the event: the shift from small groups to large groups, shifts in location, shifts in speed or intensity. For instance, we set up modes of introduction and contact that seek to intensify existing shifts in register, and then we participate in these shifts to see whether the conditions we are co-creating, with the event, make a difference in how the event unfolds and what it facilitates. It is a kind of structured improvisation that builds on an acute 
form of listening to the unfolding of a process in real time. ${ }^{1}$ Having established what we call enabling constrains, having prepared the threshold, having created the conditions for a coming-together in the mode of affirmation, we then explore how the event facilitates the creation of what Guattari called "a group subject," an ecological subjectivity in-formed by the event. There are many failures, of course, which we also take to be enabling constraints toward the future creation of techniques. Usually a failure is experienced by a resubjectifying on the figure of the individual. The problem with that is that it reestablishes the status quo, foregrounding the individual as the center of performativity rather than making the event the site of care and hospitality.

Over the years, as we have honed our practice, we have moved beyond asking how events are created (what makes an event an event) to asking what an event needs to be able to do? An event-based practice needs to reinvent itself continuously, and it needs to resist becoming institutionalized. It needs to have necessity, in the Nietzschean sense. It needs to create its own value, its own modes of evaluation. Regularly we ask ourselves whether the SenseLab still creates modes of inquiry that are productive of effects that surprise us and move us toward modes of existence that are qualitatively different from ones we have become accustomed to. Does it still produce thought? Is it still capable of making felt the thinking-in-the-moving I discussed earlier? So far, I think it is, and I believe this is largely due to the fact that we've never had a concept of membership. To be part of the SenseLab is simply to want to participate. The more you're involved, the more you affect our practices, but there is no ideal amount of involvement and no ideal time to get involved. Everything makes a difference.

ARNo BöHLER: Very beautiful, very beautiful! What you said is also important for Susanne and me. Susanne started out from the arts and is highly interested in philosophy, I started out from philosophy and I am highly interested in the arts. For us it has always been important do to both, philosophy AND art, or what we call Philosophy On Stage, a mode of arts-based philosophy. Something we are hungry for and think that it is necessary.

Today traditional academic philosophers have a lot of problems with bringing the arts together with philosophy. They think that "mixing" the arts with

1 Brian Massumi and I discuss this in more detail in our chapter "Propositions for Thought in the Act" in our forthcoming book Thought in the Act: Passages in the Ecology of Experience (Minnesota University Press). 
philosophy makes philosophy unserious. So would you - this is my last question - say something about your experience of bringing exactly art and philosophy together.

ERIN Manning: Through the SenseLab, I've found a community of people who think at the interstices of thought-in-the-making (like you and Susanne, like Brian and so many others) and I've found that it is possible, through a transdisciplinary perspective, to reinvent what it means to create in the interval of making and thinking. This has made it easier not only to overcome the closedmindedness of disciplinary boundaries but also to develop techniques that assist me in both becoming more rigorous as a philosopher and to have a more sustained practice as an artist. It's taken time because the practices are not necessarily complementary, at least not in the way they make or take time in my own experience of them. What I mean by that is that I can't easily move from writing to artmaking, or vice-versa, because their ways of making and taking time are so different. I need to create holes of time that can be populated by each practice - holes that can capture the failures in the thinking and the making that are at the heart of creating new ways of approaching each medium. So a busy back-and-forth produces only frustration. Over the years the best approach I have found is to work in chunks, spending a period of 2-3 months reading and writing, and then changing gears completely and spending time in the studio. I rarely understand the link between the two practices while I am in the making and I don't try to impose one.

One technique I am using at the moment to try to make a bridge between practices is to allow projects to develop without necessarily deciding what form they will eventually take. This involves allowing the project to investigate its own ways of generating itself before deciding how it comes to expression. In the past months, this has led me to work mostly in the studio, developing the conditions for participatory textile work, but lately it has moved me back to my desk to explore the concept of the minor gesture, a concept I am working on to define in relation to what I am thinking as the "artful" within the participatory. This is rare for me - to find a passage between writing and art where the writing actually feeds the artmaking and vice versa.

In the end, though, each practice has to be able to do its own work: the philosophy and the art have to stand on their own. And so the rigor of each practice has to be honed, and this is of course difficult with the time constraints we all have. But the SenseLab functions as a good conduit, as does the teaching I do, 
which both involve close philosophical readings paired with work in the artist studio.

Perhaps one of the most concrete ways art and philosophy come together in my practice is around the question of value. Brian and I recently did a TEDx talk called "For a Pragmatics of the Useless", where we were trying to create a vocabulary for a valuing of experience that is not allied to either capitalist marketvalue or prestige-value. Philosophy and art both sit on the side of prestige-value more than on the side of market-value. But the prestige-value obviously has market effects. Some philosophers and artists have chosen a kind of non-doing as a way of responding to the prestige-value of art and philosophy. The problem with that is that it simplifies the act, reducing it to a dichotomy. I would rather operate within a Spinozist frame, where there is an emphasis on rest in movement and movement in rest. I think that there is a lot of doing in the non-doing and a lot of non-doing in the doing. We need to keep that complexity there, focusing perhaps more on the subtleties of the in-act of autistic perception, an in-act that cannot be reduced so easily to form and category. So, instead of going that way, we proposed the reinvigoration of the concept of uselessness, emphasizing a kind of doing, an in-act, where value is not pre-inscribed but created.

I hope that both the philosophy and the art I do are useless in this sense. I think if more and more of us are actively-activistly thinking about the useless in a kind of speculatively pragmatic sense, there is a hope that we can redefine value outside of a capitalist mandate. In so doing, perhaps we'll even be able to come back to the original idea of philosophy, which had nothing to do with a discipline. Philosophy was what you did in relation to other practices, it was what emerged from practices that remained other, such as art, mathematics, physics, rather than being a field that needed to separate itself out. What if we took philosophy and art not as disciplines but as forms of thinking in the making, and making in the thinking? What if the thinking-doing were conceived as a value in itself, independent of what form it takes?

ARNo BöHLER: Thank you!

ERIN MANNING: Thank you! It was a real pleasure. 
